\title{
Entrelacs
}

ENTRELACS Cinéma et audiovisuel

12 | 2016

Nouvelles formes audiovisuelles documentaires

\section{Le versant promotionnel du webdocumentaire dans les univers fictionnels transmédiatiques}

Hélène Laurichesse

\section{(2) OpenEdition}

\section{Journals}

\section{Electronic version}

URL: http://journals.openedition.org/entrelacs/1876

DOI: $10.4000 /$ entrelacs. 1876

ISSN: 2261-5482

\section{Publisher}

Éditions Téraèdre

Electronic reference

Hélène Laurichesse, « Le versant promotionnel du webdocumentaire dans les univers fictionnels transmédiatiques », Entrelacs [Online], 12 | 2016, Online since 14 January 2016, connection on 01 May 2019. URL : http://journals.openedition.org/entrelacs/1876 ; DOI : 10.4000/entrelacs. 1876

This text was automatically generated on 1 May 2019.

Tous droits réservés 


\title{
Le versant promotionnel $\mathrm{du}$ webdocumentaire dans les univers fictionnels transmédiatiques
}

\author{
Hélène Laurichesse
}

1 Plus qu'un format ou un genre, le webdocumentaire s'impose désormais comme un concept ${ }^{1}$ à facettes multiples, sorte de "mot-valise ", «fourre-tout » rendant complexe une définition figée. Une nébuleuse qui s'illustre dans l'interrogation posée par Nicolas Bole et Cedric Mal Le webdocumentaire existe-t-il ? dans un ouvrage ${ }^{2}$ dédié à cerner le phénomène. Si littéralement, le webdocumentaire peut s'envisager comme un documentaire réalisé pour un support web, ses caractéristiques s'illustrent par la combinaison de plusieurs éléments : l'ancrage dans une réalité, une palette multimédia (vidéo, son, texte, image, etc..), une navigation non linéaire pour progresser dans le récit, une forme interactive. Parmi les formes d'expression très diverses qui intègrent ces éléments, celle du webdocumentaire "promotionnel» a retenu notre intérêt. La " promotion » à laquelle nous faisons référence est à prendre ici au sens américain, c'està-dire d'une communication marketing qui opère pour valoriser une entreprise ou une œuvre culturelle. Cette facette un peu obscure (car peu explorée) du webdocumentaire constitue pourtant l'un de ses sous-genres en forte progression. À mi-chemin entre le documentaire et la communication, deux axes peuvent être identifiés pour exercer ce type de promotion par le biais du webdoc : l'institutionnel (ou corporate) et la fiction. Le premier agit pour le compte des ONG, des fondations, des entreprises, ou des marques ${ }^{3}$ pour servir les intérêts de leur structure. Le second, qui constitue l'objet de notre analyse, s'inscrit dans le cadre du lancement d'œuvres dites transmédiatiques. Il sert alors à promouvoir la sortie d'un film, d'un livre, ou d'un jeu vidéo dans la dynamique du Transmedia Storytelling, cette forme narrative récente conceptualisée par Henry Jenkins il y a une dizaine d'années ${ }^{4}$. Elle structure le récit à partir de plusieurs éléments fictionnels disponibles sur différents médias en ambitionnant de « créer une expérience de divertissement unifiée et coordonnée»? Elle repose largement sur la notion de culture participative qui place les publics au cœur du fonctionnement du système dans une 
dynamique de co-construction des contenus. Dans ce contexte, nous montrerons que le webdocumentaire s'insère dans une dimension stratégique en tant que pièce d'un puzzle global. Nous en présenterons ensuite ses caractéristiques à partir de l'un des premiers exemples de production d'un webdocumentaire promotionnel dans le cadre du lancement de la série américaine True Blood. Nous verrons ensuite qu'à l'image de cet exemple, les dispositifs proposés dans ce cadre brouillent les frontières entre la fiction et la réalité dans une perspective promotionnelle pour le moins ambiguë, puisque précisément, elle est rarement perçue comme telle.

\section{La stratégie de l'attention et de l'engagement dans les politiques éditoriales des industries culturelles}

2 Envisager les contenus sous l'angle des stratégies revient à envisager de concert les questions artistiques et économiques dans un contexte environnemental donné. Or précisément, c'est ce contexte évolutif qui conduit à une remise en cause du modèle dominant dans les industries culturelles (selon lequel l'offre crée la demande), puisque l'économie de l'abondance, qui caractérise les marchés actuels, a conduit les entreprises à modifier leur politique éditoriale en augmentant l'intensité de leurs signaux, notamment marketing. L'économie de l'attention ${ }^{5}$ se trouve ainsi au cœur des enjeux médiatiques dans un contexte où l'étendue de l'offre (accentuée par les NTIC ${ }^{6}$ et la dématérialisation des supports) provoque une dispersion de l'intérêt. Les nouvelles générations, familiarisées avec les pratiques de multitasking, provoquent une généralisation du phénomène de zapping à tous les contenus. Ce procédé traduit une multi-activités, qui mobilise plusieurs moyens de communication en même temps : par exemple, regarder la télévision tout en répondant à des SMS sur son téléphone portable, tout en recherchant des informations sur une tablette ou un ordinateur. Sur cette base, chercher à attirer l'attention et adapter les contenus aux nouveaux comportements du public constituent des objectifs prioritaires.

3 Les narrations transmédiatiques, en l'occurrence, proposent précisément des contenus susceptibles de répondre à ces comportements et aux besoins de participation, d'immersion et d'interactions sociales qui se sont développées avec les nouvelles technologies de l'information et de la communication. L'interactivité et le processus de délinéarisation $d u$ récit qui vont de pair avec cette forme de récit permettent à l'internaute de s'approprier les contenus quand il le souhaite et dans l'ordre qui lui convient. Ce processus de narration contribue à capter l'attention et à susciter l'engagement des publics en s'appuyant largement sur le modèle immersif et ludique des jeux vidéo qui a prouvé sa performance sur ce terrain ${ }^{7}$. Jugé exemplaire pour capter l'attention, ce modèle se pose en référence pour stimuler à la fois sa durée et son intensité. La durée de l'attention s'inscrit dans une recherche de fidélisation pendant que son intensité répond à des alertes (zapping, buzz sur Internet). Ainsi, l'objectif de captation de l'attention à court terme, se complète à terme d'un enjeu d'engagement et de fidélisation rendant le procédé ludique inhérent au dispositif du webdocumentaire promotionnel. Cette dynamique de "gamification» de la création qui se traduit par "l'ajout de fonctionnalités interactives et ludiques à des contenus ${ }^{8}$ " va d'ailleurs bien audelà de la perspective promotionnelle ${ }^{9}$. Sa finalité est celle de la recherche d'attractivité : «l'ennui est l'ennemi » comme le souligne très justement Michel Reilhac ${ }^{10}$. 
4 L'exemple qui suit permet d'illustrer comment concrètement se manifestent ces dispositifs et d'envisager la place et le rôle du webdocumentaire dans cette dynamique.

\section{The « True Blood Revelation »}

5 Si l'on considère l'un des premiers exemples emblématiques de cette tendance, celui du webdocumentaire proposé ${ }^{11}$ dans le cadre du lancement de la Saison 1 de la série télévisée True Blood ${ }^{12}$ en 2008, on peut déjà y retrouver toutes les caractéristiques des lancements des fictions télévisées ou cinématographiques actuels. La vidéo intitulée «True Blood Revelation", se présente comme un documentaire, construit autour de reportages (supposés issus d'un journal d'information télévisé), ponctués par une série de témoignages et d'interviews de différents protagonistes supposés être réels (c'est-à-dire exister dans la vraie vie) alors qu'ils sont joués par des acteurs. Elle vise à ancrer la fiction dans une réalité en introduisant la forme documentaire afin de rendre l'expérience plus immersive. Le film réalisé expose le contexte qui précède l'histoire qui va être racontée au début de la Saison 1 de la série. Pour bien comprendre la démarche, il faut préciser que la série télévisée, elle-même inspirée d'une série de romans ${ }^{13}$, propose un monde dans lequel coexistent les vampires et les humains. La narration s'appuie ainsi sur l'un des ressorts principaux du Transmedia Storytelling: la fabrication d'un monde imaginaire, éloigné de la réalité, mais rendu possiblement réel par une multitude de détails inventés à cet effet. Ce qui est important avec ce type d'offres, souligne David Peyron ${ }^{14}$, c'est avant tout de pouvoir plonger dans un autre univers, qui puisse être crédible à partir d'un ensemble de détails cohérents. En cela, le dispositif du webdocumentaire apporte une forme de caution à la forme fictionnelle en introduisant des éléments qui la rendent réelle.

6 Dans l'exemple évoqué, le film prequel qui explique comment les vampires ont pu s'intégrer dans la société grâce à une boisson de sang synthétique conçue par des scientifiques, permet de comprendre que ce breuvage, en offrant aux vampires une alternative au sang humain, les dispense de tuer pour se nourrir, et permettent aux deux peuples une cohabitation sur terre. Il sert à crédibiliser la proposition du monde dépeint au début de la série télévisée à partir de témoignages des défenseurs des droits des vampires et de leurs opposants. Par ailleurs, ce film s'inscrit parmi un ensemble d'autres dispositifs médiatiques ayant le même objectif : introduire une "pseudo réalité » pour promouvoir la fiction.

7 Parmi ces dispositifs, plusieurs «vrais/faux» sites Internet sont créés : celui de l'American Vampire League ${ }^{15}$ qui soutient la cause des vampires, le site et la vidéo consacrés à L'Église de la Communauté du Soleil, (Fellowship of the Sun) ${ }^{16}$ se positionnant au contraire dans une position anti-vampires, celui d'un site de rencontres type Meetic destiné aux vampires et à ceux qui les aiment. La création d'un "vrai/faux » journal annonçant l'existence des vampires le «New York Announceur» vient aussi renforcer l'ancrage du récit fictionnel dans le réel.

8 Le dispositif du lancement de la Saison 2 s'appuiera, dans la même logique, sur une vaste campagne d'affiches publicitaires (ci-dessous les exemples de la marque Mini et Gillette) proposant de vrais produits et marques directement adressées à une cible fictive (ou fictionnelle), celle des vampires, et à une cible bien réelle, celle des fans de la série télévisée et de l'univers de True Blood. 


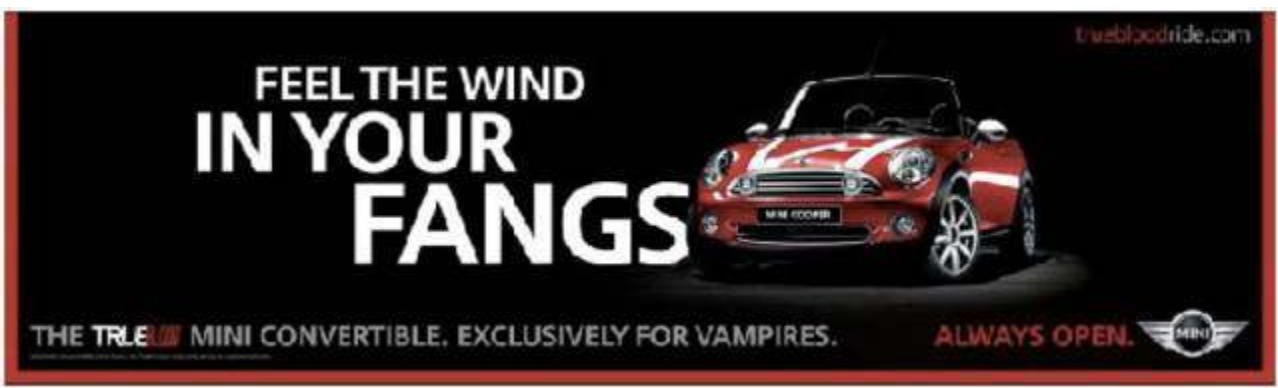

Figure 1 : Une mini cooper exclusivement pour les vampires

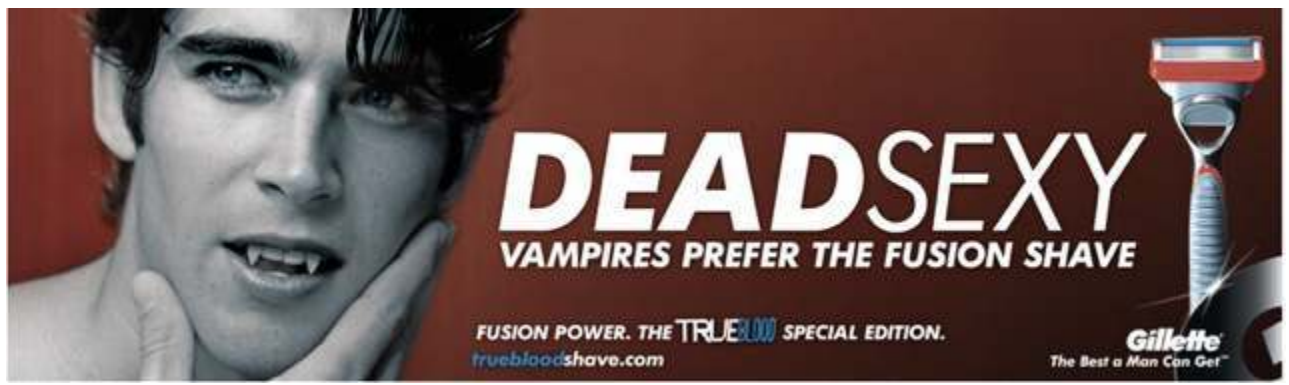

Figure 2 : Une édition spéciale des rasoirs Gillette destinée aux vampires

\section{Une pseudo-réalité pour une forme promotionnelle ambiguë}

Les contenus des webdocumentaires promotionnels tendent à fournir des éléments tangibles pour alimenter un monde parallèle (au nôtre) inventé dans une fiction. La forme promotionnelle qui en découle repose sur un trouble de la réalité comme en attestent les désormais nombreux exemples qui parsèment la grande majorité des fictions américaines (séries TV ou films) et une majorité croissante de séries télévisées françaises ${ }^{17}$.

10 Les dispositifs mêlant réalité et fiction s'illustrent notamment à travers les jeux en réalité alternée qui combinent des épreuves virtuelles sur le net et physiques dans la vraie vie (rechercher des indices dans sa propre ville par exemple). Le webdocumentaire promotionnel constitue le plus souvent un des éléments (parmi d'autres) de ces ARG. On peut aussi citer l'Advergaming qui consiste à créer un jeu plutôt que de faire un placement de marque dans un jeu existant, et également la création de chaînes de télévision ou de journaux dédiés à la promotion.

11 Le principe de gamification évoqué précédemment contribue précisément à mêler le réel et la fiction, le « vrai » et le «faux » pour assurer l'immersion nécessaire au processus. Le webdocumentaire apporte en ce sens une caution du « réel» qui vient contribuer à crédibiliser le monde fictionnel des récits transmédiatiques. Tout en n'échappant pas plus que le documentaire aux questionnements sur son rapport imparfait au réel, puisqu'il n'en est qu'une construction ou une interprétation, le webdocumentaire promotionnel constitue une passerelle attractive pour réaliser le rapprochement des frontières entre la réalité et la fiction. Il vient cautionner, crédibiliser un univers ou un récit à partir d'un traitement emprunté à celui du documentaire dans lequel des acteurs de fiction jouent à être de « vrais » personnages supposés issus du réel. 

(concepteur, participant). Il n'y a donc ni arnaque, ni tromperie, simplement un jeu où on fait semblant d'y croire, comme on peut jouer, enfant, à se faire peur. «Est-il important dans une démarche ludique généralisée de savoir distinguer le vrai" du "faux" ? N'est-il pas plus fun, plus stimulant de ne pas chercher à démêler la fiction imaginée du flux des faits réels?» Ces questions que pose Michel Reilhac ${ }^{18}$ pointent finalement les contours de ce qui serait un faux problème, et le peu de pertinence de ce type d'interrogation. D'autant que le mélange entre fiction et réalité s'exprime depuis déjà plusieurs décennies à travers les docu-fictions qui tendent à présenter des films joués par des acteurs pour des documents qui sont vrais ou encore des fictions de plus en plus souvent inspirées de faits réels. Ce qui est en revanche plus nouveau, c'est la difficulté à identifier à partir de ces contenus hybrides si l'on a à faire à du marketing ou non. Ce qui est assez nouveau également, c'est que la promotion des fictions prenne une forme documentaire.

Ces contenus, à mi-chemin entre l'artistique et le commercial, ravivent la polémique entre deux visions du transmédia qui s'opposent : l'une s'appuyant sur des créations pensées dès l'origine du projet dans une vision pure artistique, l'autre sur une forme d'opportunisme visant à exploiter le succès d'une œuvre sur un média donné sur de nouvelles plateformes.

14 Cette dernière approche, aujourd'hui dominante dans les industries culturelles, traduit la tendance d'un double mouvement à l'œuvre : celui de la culture qui se «marchandise » et celui des marques qui se «culturisent ». Un paradoxe naît de ces déplacements avec un marketing qui tend à s'effacer derrière des contenus qui ne sont plus identifiables en tant que tels tout en devenant omniprésents par un effet cumulatif des anciens dispositifs toujours à l'œuvre (la publicité notamment) et des nouveaux qui viennent s'y juxtaposer. Karine Berthelot Guiet, Caroline Marti de Montety et Valérie Patrin-Léclère parlent à ce sujet de "dépublicitarisation" et "d'hyperpublicitarisation » ${ }^{19}$ pour traduire ce brouillage énonciatif généralisé de discours s'immisçant dans toutes les sphères de la société.

15 Ces réflexions soulignent la difficulté à cerner et cloisonner aujourd'hui des notions qui auparavant ne présentaient pas de réelle ambiguïté : le marketing, la promotion, la création. À présent, il apparaît que ce nous appelons ici «webdocumentaire promotionnel » pourrait tout aussi bien correspondre à la définition d'un contenu de marque : le «Brand Content».

16 «Le Brand Content désigne le fait qu'une marque crée ou édite du contenu [..] informatif, culturel, pratique, ludique ou divertissant ${ }^{20}{ }_{\text {». }}$

Regroupant des contenus informatifs, de divertissements ou de services, ces contenus prennent différentes formes: websérie, webshow, webdocumentaire, courts métrages, vidéos interactives, sites éditoriaux, expérientiels ou utilitaires, livres, applications Facebook, applications pour mobiles et tablettes. De là, si l'on admet que certains contenus transmédiatiques présentent assez largement les mêmes caractéristiques que celles des marques ${ }^{21}$ (Harry Potter, Twilight, Hunger Games), alors, les webdocumentaires de ces univers fictionnels peuvent en effet se définir comme un contenu de marque.

18 D'une certaine façon, ce type de contenus évoque la problématique ancienne des pratiques commerciales clandestines ${ }^{22}$ et celle actualisée de ce que l'on appelle aujourd'hui le marketing furtif ou stealth marketing (du nom de la technologie stealth qui rend certains bombardiers indétectables pour les radars). Il s'agit en l'occurrence de 
stratégies qui permettent de communiquer sans que l'origine du message puisse être identifiée par les destinataires ${ }^{23}$, une tendance désormais structurelle dans la discipline marketing contemporaine.

Pour autant, pour des observateurs avertis, la dimension promotionnelle de ces dispositifs promotionnels apparait finalement de manière presque flamboyante puisque les contenus concernés sont conçus à l'occasion du lancement promotionnel d'un contenu principal (le plus souvent un film). En dépit des brouillages énonciatifs évoqués, la dominante «marketing » de ces éléments de la narration transmédiatique reste tout de même plutôt évidente puisqu'ils n'existent que pour servir ce contenu principal (film, livre) lors de son lancement. En dehors de cette fonction, ces productions, envisagées de manière autonome, ne présentent pas un intérêt majeur. On notera également que ce sont des instances marketing qui récompensent leur créativité24 ${ }^{24}$

\section{Conclusion}

Nous avons souhaité apporter, dans le cadre de ce numéro sur les enjeux des nouvelles formes audiovisuelles documentaires, une réflexion à dominante stratégique sur la dimension promotionnelle du webdocumentaire, plus exactement dans le cadre des univers fictionnels transmédiatiques. Sous cet angle d'analyse, le webdocumentaire promotionnel participe, à la façon d'une pièce de puzzle, à susciter l'attention et l'engagement des publics, en s'appuyant sur une (fausse) réalité pour servir un monde fictionnel. Dans ce contexte, il va permettre de le crédibiliser, en lui apportant une forme de caution qui favorise l'immersion.

La place et le rôle du webdocumentaire promotionnel ainsi décrit soulèvent deux questionnements interdépendants : celui de la perception de la forme promotionnelle de ces dispositifs (est-il utile, important de savoir s'il s'agit de promotion ou de création?) et celui de l'utilisation d'une pseudo réalité pour promouvoir un monde fictionnel («jouer » à la réalité finalement, faire semblant d'y croire).

Eu égard au premier questionnement, nous avons déployé une argumentation dialectique en soulignant à la fois le déplacement du paradigme à l'oeuvre dans la discipline marketing et une forme d'assimilation et d'appropriation de ces nouveaux dispositifs par les publics.

23 En ce qui concerne le second questionnement, nous avons mis en avant la logique de gamification qui se généralise dans l'approche du webdocumentaire (promotionnel ou non), et l'importance à considérer l'environnement contextuel dans les choix de production et par association, dans les stratégies de politiques éditoriales. La dynamique du jeu vient répondre à aux nouvelles contraintes et conditionne, dans une certaine mesure, la nature des créations envisagées.

24 À l'issue de cette réflexion, il nous apparaît que ces nouvelles formes créatives provoquent finalement une chaîne de questionnements multiples, assortis d'une incessante remise en cause des concepts existants. Est-ce que ce que nous avons appelé webdocumentaire promotionnel correspond vraiment à de la promotion? Est-ce par ailleurs aussi une œuvre au sens artistique ? Est-ce que l'approche narrative globalisante que nous avons décrite est vraiment du transmédia? Autant de questions qui pourraient se prolonger et se démultiplier en fonction d'autres angles d'approches, d'autres problématiques : on pourrait se demander par exemple si les créations qui entrent dans le 
registre du webdocumentaire appartiennent vraiment à la famille du documentaire, si ce que nous appelons «publics » correspond encore au terme de référence tant le rôle et la place qu'ils occupent se sont modifiés, etc.. Le foisonnement de néologismes qui émergent actuellement caractérise une situation instable, puisqu'en constante adaptation vis-à-vis de son environnement. Les interprétations multiples et les formes d'appropriation qui en découlent sont le résultat de la nature hybride de ces contenus et du manque de recul dont nous disposons sur ces phénomènes.

Ce qu'il est intéressant de souligner dans cette dynamique, c'est la nécessité à privilégier la relation à long terme avec le public plutôt que la transaction à court terme. La rentabilité des offres proposées en l'occurrence, ne constitue pas un impératif prioritaire puisque le webdocumentaire promotionnel (tout comme les autres éléments de la narration transmédiatique) est proposé en accès gratuit. On voit bien que ce qui se dessine n'est pas une simple manœuvre instrumentale et bassement commerciale, il s'agit plus profondément de reconstituer un nouvel écosystème dans le fonctionnement des industries culturelles et médiatiques.

\section{BIBLIOGRAPHY}

Bibliographie

Berthelot Guiet K. Paroles de pub, la vie triviale de la publicité, Éditions non standard, Saint-Romain de Colbosc, 2013.

Berthelot-Guiet K., Marti de Montety C., Patrin-Leclère V. La fin de la publicité ?, Le bord de l'eau, Lormont, 2014.

Bô D. 2012. Du Brand Content à la Brand Culture. Les Clefs d'une stratégie de contenu, Vol 2.

QualiQuanti, Paris. [en ligne] http://www.leslivresblancs.fr.

Bole N. et Mal C., Le webdocumentaire existe-t-il ?, Lulu Press, 2014.

Boullier D., « Les industries de l'attention : fidélisation, alerte ou immersion »,Réseaux, Vol. 2, n 154,2009 , p. 231-246.

Goldhaber M.H., " The Attention Economy and the Net », First Monday, Vol.2, n²4, 1997.

Jenkins H, Convergence Culture: Where Old and New Media Collide, New York University Press, 2006.

Laurichesse H., (dir.), La stratégie de marque dans l'audiovisuel. Armand Colin Recherches, Paris, 2013.

Laurichesse H., Quand les films deviennent des marques, Au croisement de la "culturalisation » des marques et du branding des industries culturelles. Habilitation à Diriger les Recherches, Université Toulouse Jean-Jaurès, 2 Avril 2015.

Packard V., La persuasion clandestine, Calman Levy, 1957. 
Pedon E., Le web documentaire : dispositifs et enjeux d'une pratique en devenir in Morelli P., PignardCheynel N. et Baltazart D., (Dir.), Actes du Colloque EUTIC : Publics et pratiques médiatiques, Université de Lorraine, Metz, 17-19 Octobre 2012, p. 217-227.

Peyron D., « Quand les œuvres deviennent des mondes, Une réflexion sur la culture de genre contemporaine à partir du concept de convergence culturelle ", Réseaux, Vol.26, n 148-149, 2008, p. 335-368.

Reilhac M., «Quand les récits transmedia se préparent à ré-enchanter notre quotidien », [en ligne] http://www.lemonde.fr/idees/article/2010/07/02/quand-les-recits-transmedia-sepreparent-a-re-enchanter-notre-quotidien_1382030_3232.html

Simon H., « Designing Organizations for a Information-Rich World » in Greenberger M. (Dir.) Computer, Communications and The Public Interest, John Hopkins Press, Baltimore, 1971.

\section{NOTES}

1. Pedon E., Le web documentaire : dispositifs et enjeux d'une pratique en devenir in Morelli $\mathrm{P}$; Pignard-Cheynel N et Baltazart D (Dir.), Actes du Colloque EUTIC: Publics et pratiques médiatiques, Université de Lorraine, Metz, 17-19 Octobre 2012, p. 217-227.

2. Bole N. et Mal C., Le Webdocumentaire existe-t-il ?, Lulu Press, 2014.

3. Médecins dans Frontières, Association sœur Emmanuelle, Enfants du Mékong, la Fondation Abbé Pierre, la Croix-Rouge, WWF, le ministère des Affaires étrangères (http:// destinations.diplomatie.gouv.fr), SFR (Homo Numericus : une série de portraits destinés à faire comprendre le monde numérique).

4. Jenkins H., Convergence Culture: Where Old and New Media Collide, New York University Press, 2006, p. 95-96.

5. Simon H., « Designing Organizations for a Information-Rich World » in Greenberger M. (Dir.) Computer, Communications and The Public Interest. John Hopkins Press, Baltimore, 1971. Goldhaber M.H. "The Attention Economy and the Net », First Monday, Vol.2, n4 1997.

6. Nouvelles Technologies de l'Information et de la Communication.

7. Boullier D., « Les industries de l'attention : fidélisation, alerte ou immersion ", Réseaux, Vol. 2, n 154, p. 231-246, 2009.

8. Guillaud H., « MasterClass Internationale du Transmedia », Marseille, 11, 12, 13 Mai 2011. [en ligne] http://www.internetactu.net/2011/05/18/transmedia-12-laconvergence-des-contenus/.

9. Le néologisme de « gamedocumentaire » illustre d'ailleurs cette tendance.

10. Reilhac M., «Quand les récits transmedia se préparent à ré-enchanter notre quotidien », 2010.[en ligne] http://www.lemonde.fr/idees/article/2010/07/02/quand-lesrecits-transmedia-se-preparent-a-re-enchanter-notre-quotidien_1382030_3232.html.

11. [en ligne] http://www.youtube.com/watch?v=RE0Z_MP7bBA True Blood : Revelation.

12. Autres exemples : «Why So Serious » a été conçu pour promouvoir The Dark Night , «The Beast » pour promouvoir AI.

13. « La communauté du Sud » de Charlaine Harris, treize tomes. 
14. Peyron D. «Quand les œuvres deviennent des mondes, Une réflexion sur la culture de genre contemporaine à partir du concept de convergence culturelle ", Réseaux, vol.26, n 148-149, 2008, p.335-368.

15. Aujourd'hui supprimé, il proposait des d'articles, des vidéos, de films préventifs et témoignages d'anciens junkies accros au « $\mathrm{V}$ », une drogue illégale qui n'est autre que du sang de vampire.

16. [en ligne] https://www.youtube.com/watch?v=9cw_Bw6J5bA.

17. Braquo, Plus belle la vie, Engrenages, Les Témoins ...

18. Reilhac M., ibid.

19. Berthelot-Guiet K., Marti de Montety C., Patrin-Leclère V. La fin de la publicité ?, Le bord de l'eau, Lormont, 2014.

20. Bô D., Du Brand Content à la Brand Culture. Les Clefs d'une stratégie de contenu, Vol 2. QualiQuanti, Paris. p.3. 2012. [en ligne] http://www.leslivresblancs.fr.

21. Laurichesse H., (dir.), La stratégie de marque dans l'audiovisuel. Armand Colin Recherches, Paris, 2013, Laurichesse H., Quand les films deviennent des marques, Au croisement de la "culturalisation" des marques et du branding des industries culturelles. Habilitation à Diriger les Recherches, Université Toulouse Jean-Jaurès, 2 Avril 2015.

22. Packard V., La persuasion clandestine, Calman Levy, 1957. La théorie de la manipulation a ensuite été étudiée dans les années 60 par de nombreux chercheurs dans des champs disciplinaires variés, notamment, Alfred Marshall, John Kenneth Galbraith, Adorno et Horkeimer, Habermas, Marcuse. Voir à ce sujet Berthelot Guiet K. Paroles de pub, la vie triviale de la publicité, Éditions non standard, Saint-Romain de Colbosc, 2013.

23. L'exemple désormais célèbre de la famille Jones illustre parfaitement le principe d'une étendue infinie de possibilités à développer pour pratiquer le marketing de manière clandestine. Le clip qui illustre le procédé met en scène une (fausse) famille, représentée par David Duchovny et Demi Moore et leurs deux enfants, qui emménage dans un quartier résidentiel avec l'objectif de vendre un maximum de produits. Représentant la famille parfaite, leur rôle consiste à rendre leurs voisins si envieux de leur mode de vie que pour leur ressembler, ils vont se mettre à acheter les mêmes produits et marques qu'eux.

24. Le jeu en réalité alternée "Why So Serious » a obtenu le Grand Prix du Cannes Lions Festival par exemple.

\section{ABSTRACTS}

This article considers webdocumentary strategics issues as piece of a transmedia promotionnel puzzle in the field of fiction. Estimate his place and role in a convergence culture involve to think webdocumentary in an editorial policy perspective. We show the webdocumentary promotional and playful aspects in an attention economy approach. In a gamification process, he mix up fiction and reality in order to allow audiences commitment and immersion. 
Cette communication vise à appréhender les enjeux stratégiques du webdocumentaire en tant qu'outil promotionnel d'un univers fictionnel transmédiatique. Sous l'angle de la problématique de l'attention, le webdocumentaire correspond à un modèle ludique et promotionnel. Dans un processus de gamification, il entremêle fiction et réalité pour permettre l'engagement et l'immersion des publics.

\section{AUTHOR}

\section{HÉLÈNE LAURICHESSE}

Maître de conférences, HDR, à l'ESAV (Ecole Supérieure d'Audiovisuel) de l'Université Toulouse Jean-Jaurès. Auteure des ouvrages « Quel marketing pour le cinéma? » publié aux éditions du CNRS en 2006, et « La stratégie de marque dans l'audiovisuel » aux éditions Armand Colin, ses recherches portent sur les stratégies marketing et économiques dans le secteur audiovisuel. 